\title{
Study of d.c. electrical conductivity of paranitroaniline doped $(1: 1)$ polyvinylchloride and poly(methyl methacrylate) polyblends
}

\author{
R V WAGHMARE*, N G BELSARE, F C RAGHUWANSHI and S N SHILASKAR ${ }^{\dagger}$ \\ Department of Physics, Vidya Bharti Mahavidyalaya Camp, Amravati 444 602, India \\ ${ }^{\dagger}$ Department of Electronics, Vishwakarma Institute of Technology, Pune 411 037, India
}

MS received 8 September 2006; revised 8 January 2007

\begin{abstract}
Electrical properties of PVC, PMMA and their $1: 1$ polyblends, before and after adding paranitroaniline into them, have been investigated as a function of temperature, electric field and dopant concentration, to study the mechanism of electrical conduction. The current was measured by applying d.c. voltage in the range $25-800 \mathrm{~V}$ at various thermostatically controlled temperatures $(313-373 \mathrm{~K})$. The results obtained predict the Schottky-Richardson conduction mechanism to be operative and d.c. conductivity of the blend lies intermediate between those of individual components. Further, the conductivity of the blend increases with temperature and applied electric field and also with the increase in concentration of dopant. To identify the mechanism governing the conduction, the activation energies in low temperature (LTR) and high temperature (HTR) regions have been calculated. The dielectric constant of the sample at various temperatures have been calculated which increased with increase in temperature. This is indicative of the diffusion of ions in space charge polarization at higher temperature. The study of XRD and FTIR supports the changes occurring in the conductivity of the blend.
\end{abstract}

Keywords. Paranitroaniline; polyvinylchloride; poly(methyl methacrylate).

\section{Introduction}

The physical mixing or blending of two polymers produces an alloy with quite different properties, which can be potentially useful (Bahadur and Sastry 2003). The d.c. electrical conductivity studies are aimed at understanding the origin of the charge carrying species, their numbers and the way in which they move through the bulk of the material. These parameters are related to the chemical composition, microstructure and morphology of the material (Seanor 1982). The wide spread use of PVC is attributed mainly to its high electrical and chemical resistance and its unique ability to be mixed with additives to produce a large number of compounds with wide range of physical and chemical properties (Smith 1990). Despite the polar nature of polyvinylchloride (PVC), it is used at large scale as an insulating material because of the constancy of its dielectric properties. Poly(methyl methacrylate) (PMMA) is a hard, rigid, transparent thermoplastic, which has good outdoor weatherability and is more impact resistant than glass. PMMA is weakly polar (Blythe 1979). When it is blended with PVC, the dielectric constant shows no large variation with temperature in the maximum-use tempera-

*Author for correspondence (rvwaghmare@rediffmail.com) ture range. In the present investigation, d.c. conductivity of paranitroaniline(PNA) doped PVC, PMMA and their blend was measured to identify the mechanism of electrical conduction. The dielectric constants have been measured for different temperatures. Activation energies in low and high temperature regions have also been calculated.

A good number of reports (Khare et al 1994; Bahri and Seth 1997; Belsare and Deogaonkar 1998a) on the theory of electrical conduction and experimental findings have appeared in a number of such blends. The conductivity studies on PVC/PMMA polymer blend electrolytes have shown that the polymer component would render stability (Rajendran and Uma 2000). Polymers like PVC and PMMA being essentially insulating materials, the number of free charge carriers is very small and their mobility is very low. In an electric field, it is expected that a redistribution of charges that are mobile enough to respond to the time scale of the applied field, may occur. Chloro group in PVC acts as an electron-withdrawing group, which pulls electrons from neighbouring atoms, and hence tends to become a more reactive compound. PMMA containing electron releasing methyl group supplies electrons. PMMA also contains acrylate group $(=\mathrm{C}-\mathrm{CO}-\mathrm{O}-\mathrm{R})$, which is a bulky side group. Methyl substituents in the polymer decrease the $\mathrm{C}-\mathrm{C}$ bond stability. It is these considerations that led us to study the d.c. electrical conduction in PVC/PMMA blends of both undoped and doped forms. 


\section{Experimental}

PMMA (Otto kemi, Mumbai) and PVC (commercial grade) in equiweight proportions were dissolved in a solvent (cyclohexanone, SD Fine Chemicals, Mumbai). The dopant paranitroaniline (LOBA Chemie, Mumbai) was dissolved in 1,4 dioxan. The dopant and the polymer mixture were mixed together in solution form. For complete dissolution, solution was kept in a stand for 3 days. The solution mixture was poured on a perfectly plane glass plate kept floating freely in a pool of mercury for perfect leveling. It was, thereafter, allowed to evaporate in air at room temperature. Further, it was dried for $48 \mathrm{~h}$ to remove any traces of solvent.

The dry film (Belsare and Deogaonkar 1998b) was removed from the plate and cut into pieces (samples) of desired size, which were then coated on both sides with silver paint (Eltecks Corporation, Bangalore). The films of other samples were prepared by the same method. High voltage power supply, EHT-11 and pico-ammeter, DPM111 (supplied by Scientific Equipment, Roorkee) were used for $I-V$ measurements. The film was kept between the electrodes of specially designed sample holder. MASTECH k-type (MS 6500) digital thermometer was used to measure the temperature. The thickness of the film was measured by DIGMATIC micrometer (Mitutoyo Corporation). The capacitance of the film was measured using CIE-15 capacitance measuring meter. From the values of capacitance, $C$, the dielectric constants were calculated. The XRD and FTIR spectra of the samples were obtained from SAIF, Punjab University, Chandigarh. The $I-V$ characteristics for the films of PVC/PMMA blend, with and without doping were studied.

\section{Results and discussion}

The samples whose electrical conductivity have been studied include: (i) PVC $0 \%, 2 \%, 4 \%, 6 \%$, and $8 \%$ PNA doped films, (ii) PMMA $0 \%, 2 \%, 4 \%, 6 \%$, and $8 \%$ PNA doped films and (iii) PVC/PMMA $0 \%, 2 \%, 4 \%, 6 \%$, and $8 \%$ PNA doped films. The range of values of electrical conductivity for all the samples along with their comparative estimate is given in $\$ 4$.

Addition of PVC to PMMA in $1: 1$ proportion produces a blend matrix in which there are $-\mathrm{Cl}$ as electron withdrawing group and $-\mathrm{CH}_{3}$ as electron releasing group. This situation may lead to the formation of charge transfer complex resulting in the increase in conductivity over that of PMMA (but lower than that of PVC). The increase in dopant (PNA) concentration is found to increase the conductivity of the blend. This may be attributed to delocalization of electrons in the heterocyclic benzene ring which forms nitrogen dioxide ion and that increases conductivity (Francise 2003). The orientation of dipoles at elevated temperatures readily takes place under the influ- ence of electric field, which results in the releasing of charge carriers. This phenomenon is manifested in terms of increase in conductivity.

\subsection{Study of conduction mechanisms from graphs}

In the present study, the overall features of the graphs for all the samples have quite similar nature, therefore, only representative graphs for sample PVC/PMMA/4\%PNA have been included in the script.

3.1a Poole-Frenkel plots (figure 1): The $I-V$ relationship for $\mathrm{P}-\mathrm{F}$ effect is expressed as

$$
J=B \exp \left(-\phi / k T+\beta_{\mathrm{PF}} E^{1 / 2}\right),
$$

where $B$ is a constant and other symbols have their usual meanings.

The $\mathrm{P}-\mathrm{F}$ mechanism predicts a field dependence for the conductivity and is expressed as

$$
\sigma=\sigma_{0} \exp \left(\beta_{\mathrm{PF}} E^{1 / 2} / 2 k T\right)
$$

Nature of the graph $\ln (\sigma)$ vs $\sqrt{ }(E)$ indicates that the lines are almost parallel to $E^{1 / 2}$ axis. Thus, it is inferred that the contribution due to $\mathrm{P}-\mathrm{F}$ mechanism to conduction is not very significant.

3.1b Schottky plots (figure 2): Schottky emission occurs due to thermal activation of electrons over metal-insulator interface. The applied electric field further reduces the height of the barrier. The Schottky-Richardson currentvoltage relationship is expressed as

$$
J=A T^{2} \exp \left(-\phi_{\mathrm{s}} / k T+\beta_{\mathrm{SR}} E^{1 / 2}\right),
$$

and hence

$$
\log J=\log A T^{2}-\phi_{\mathrm{S}} / k T+\beta_{\mathrm{SR}} E^{1 / 2} .
$$

If we plot $\ln (J)$ vs $E^{1 / 2}$, we obtain a straight line for higher voltages $(>100 \mathrm{~V})$. This line is known as Schottky line.

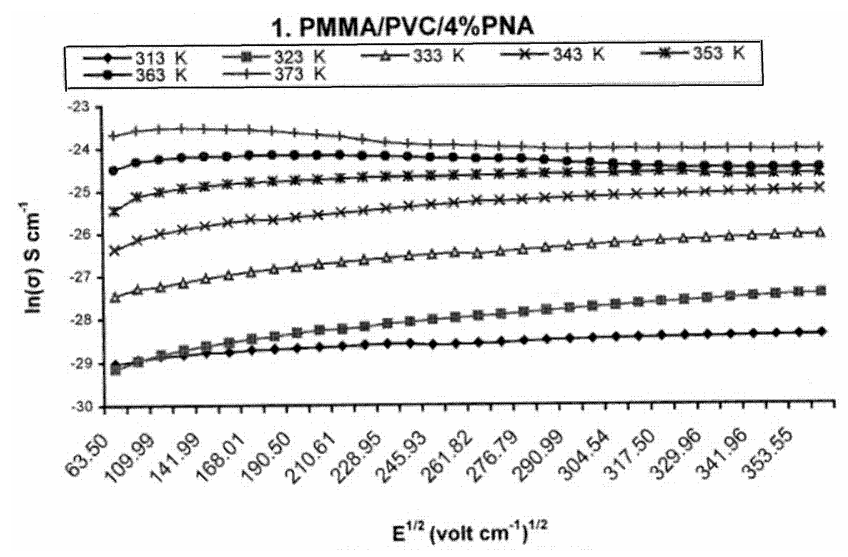

Figure 1. Poole-Frenkel plots for PVC/PMMA/4\%PNA. 
In the present case we get such lines in the graph of figure 2, which indicate the existence of Schottky mechanism.

3.1c Arrhenius plots (figure 3): The electrical conductivity can be expressed as

$$
\sigma=\sigma_{0} \exp \left(-E_{\mathrm{a}} / k T\right),
$$

where $E_{\mathrm{a}}$ is activation energy. Thus, when we plot $\ln (\sigma)$ vs $1 / T$, a straight line with a negative slope is obtained. For the present blend, these plots show straight lines with negative slopes indicating the temperature dependence of conductivity.

\subsection{Activation energy}

The values of activation energies have been calculated from the Arrhenius plots using the equation

$$
\sigma=\sigma_{0} \exp \left(-E_{\mathrm{a}} / k T\right) \text {. }
$$

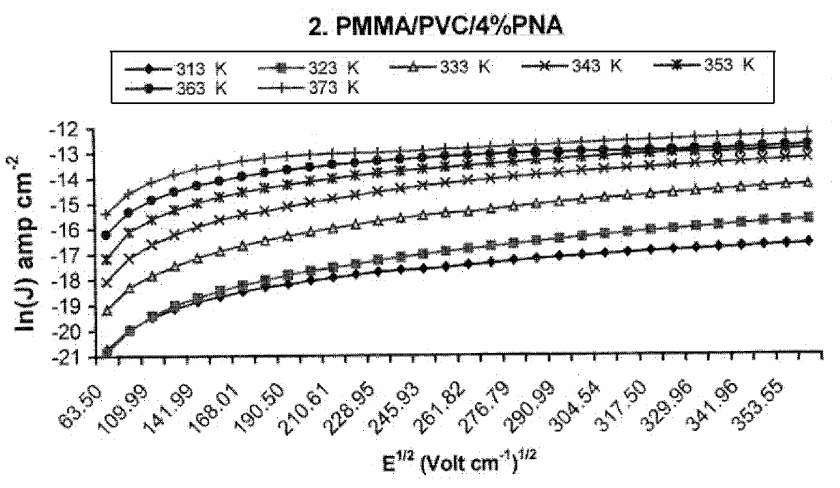

Figure 2. Schottky plots for PVC/PMMA/4\%PNA.

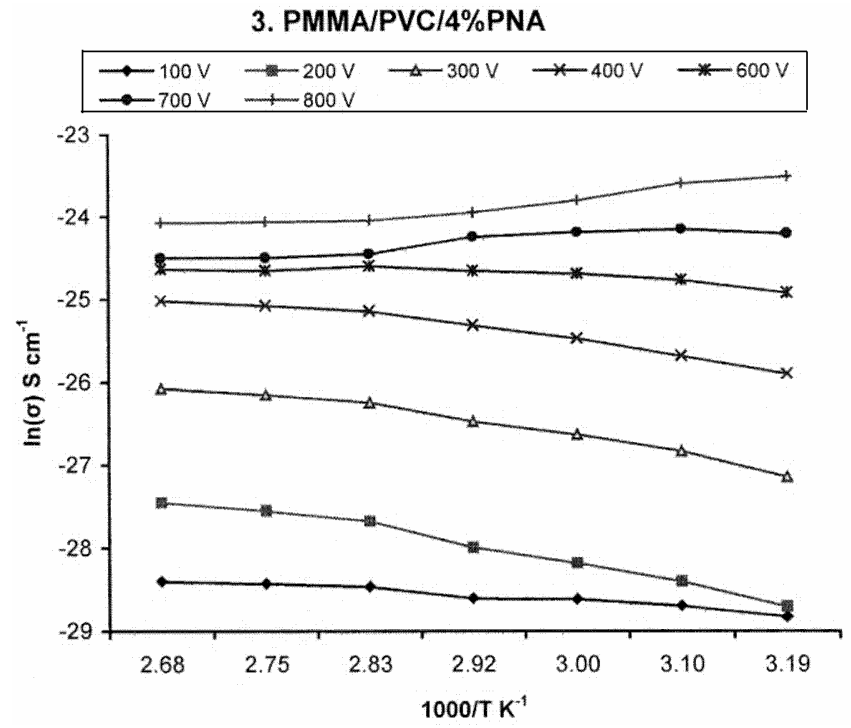

Figure 3. Arrhenius plots for PVC/PMMA/4\%PNA.
For PMMA, when voltage is increased from $200-300 \mathrm{~V}$ in LTR, $E_{\mathrm{a}}$ increases from $0.84-0.85 \mathrm{eV}$ and further as voltage increases from $400-800 \mathrm{~V}, E_{\mathrm{a}}$ decreases continuously. For PVC, $E_{\mathrm{a}}$ decreases from $0.35-0.13$ for the voltage range $200-400 \mathrm{~V}$ and then it changes from $0 \cdot 28-0 \cdot 38$ for 600-800 V.

In most of the cases the values of $E_{\mathrm{a}}$ in the blend lie intermediate between those of PVC and PMMA individually in LTR and HTR. In HTR, for almost all the samples, the values of $E_{\mathrm{a}}$ decrease from $0.70-0.34$ almost continuously with the increase of electric field at constant temperature.

At any temperature only a fraction of molecules or atoms in the system will have sufficient energy to reach activation energy level of $E_{\mathrm{a}}$. As the temperature of the system is increased, more and more molecules or atoms will attain the activation energy level. At high temperature, the activation energy of dipole segmental processes decreases due to disturbance of the co-operative movement of segments. This explains the decrease of activation energy with increase in temperature.

The only marginal increase in conductivity of the blend matrix with the increase of dopant concentration can be chiefly attributed to the localization of charge carriers. There are two factors (Skotheim 1986) which encourage this localization: (i) electron-lattice interaction and (ii) disorder. The electrons, which are injected by the electrodes, lead to electron-lattice interaction, which arises from the dependence of the wave function and the energy of charge carriers on the positions of the molecules of the solid. This interaction may lead to the development of cooperative segmental movements about a carrier that lowers its energy (Tager 1972).

The potential energy of the injected charge can be reduced by the displacement of the side group about it. These displacements of side chains produce a sufficiently deep potential well in which excess charge can occupy a bound state. This explains a very marginal variation of conductivity in case of PNA doped sample w.r.t. temperature and electric field as seen from the Arrhenius plots. The decrease in activation energy with temperature and electric field suggests the delocalization of charge carriers as a result of increase in temperature and electric field.

\subsection{Behaviour of dielectric constant}

The values of dielectric constant, $\varepsilon_{\mathrm{T}}$, of PMMA, PVC, PVC/PMMA, PVC/PMMA/4\%PNA and PVC/PMMA/ $8 \% \mathrm{PNA}$ in the temperature range $313-373 \mathrm{~K}$ vary in the range $4 \cdot 88-6 \cdot 27,3 \cdot 45-7 \cdot 67,3.06-5 \cdot 34,4.33-7.71$ and $7 \cdot 73-13 \cdot 37$, respectively.

It is observed that for all the sample films, the dielectric constants increase with the increase of temperature and also with the concentration of the dopant. This is justified from the consideration of the effect of temperature. The 
temperature facilitates the diffusion of ions in the space charge polarization. Thermal energy may also aid in overcoming the activation barrier for the orientation of polar molecules in the direction of the field. However, on melting, the molecules will have sufficient thermal energy to orient themselves in the applied field. Correspondingly, the relative dielectric constant increases. When applied field was increased, it was found that the dielectric constants remained almost same. The relatively large polar molecules are not able to orient themselves in the solid state under the influence of an electric field. Therefore, dielectric constant remains almost the same with increasing electric field.

The measurement of dielectric constant $\left(\varepsilon_{\mathrm{r}}\right)$ reveals that the value of $\left(\varepsilon_{\mathrm{r}}\right)$ for the blend sample is intermediate between that of PVC and PMMA individually at almost all temperatures. However, the addition of dopant PNA increases the value of dielectric constant. This may be attributed to the polar nature of PNA. The PNA being an amine, dipoledipole interaction (specially hydrogen bonding) is present in it. Primary amines can participate in intermolecular hydrogen bonding. The resonance forms of PNA show that the nitro group increases electron delocalization as

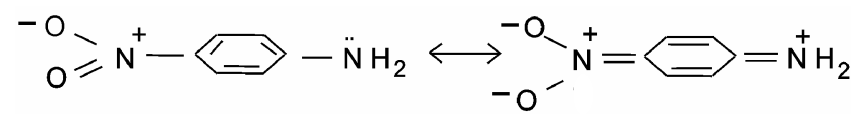

PNA is a well known second order nonlinear optical material (Purohit and Joshi 2001) in which one donor, $\mathrm{NH}_{2}$ and one acceptor, $\mathrm{NO}_{2}$ are located at the opposite ends of the conjugated system. The substituents donate or accept $\pi$ electrons from the skeletal benzene ring, thereby, removing the centre of symmetry. The electrical properties are influenced more by dipole asymmetry than by the presence of polar groups. When the dipoles are able to respond readily to changes in temperature and electric field, the dielectric constant is high. This explains the increase in dielectric constant of the blend with the addition of PNA.

In the absence of any thermal excitation, the influence of voltage stress is usually less marked than other factors. This means that the effect of temperature is remarkable. Even in the absence of electric field, the temperature alone can cause orientation of the dipoles in some common direction. Thus the temperature causes to increase the dielectric constant of PNA doped blends.

In order to be of use in electrical and electronic applications, a plastic should have reasonably constant dielectric properties over a broad range of temperatures, frequencies and humidity. For general insulating or protective coating, dielectric constant up to 8 may be acceptable but maximum dielectric constant (Goosey 1985) specified by MIL-I16923 for material used in electronic and electrical assemblies is 4.5 at $1 \mathrm{kHz}$.

In the present study it is observed that the value of $\varepsilon_{\mathrm{r}}$ for (PVC/PMMA) blend varies from 3.09-5.34 and for PVC/PMMA/4\% PNA blend from 4.33-7.11 over a large temperature range from $313-373 \mathrm{~K}$. It indicates that doping of PNA up to $4 \%$ in PVC/PMMA blend may serve the purpose in electrical insulating applications. As against these, with $8 \%$ doping the $\varepsilon_{\mathrm{r}}$ varies very much in between 7.73 and 13.57 indicating unsuitability for insulating applications. This is in agreement with the literature (Goosey 1985).

\subsection{FTIR study}

In the PVC/PMMA/4\% PNA blend, the absorption bands are broadened over the frequency range $2940-1146 \mathrm{~cm}^{-1}$ due to the addition of dopant (figure 5). A single absorp-

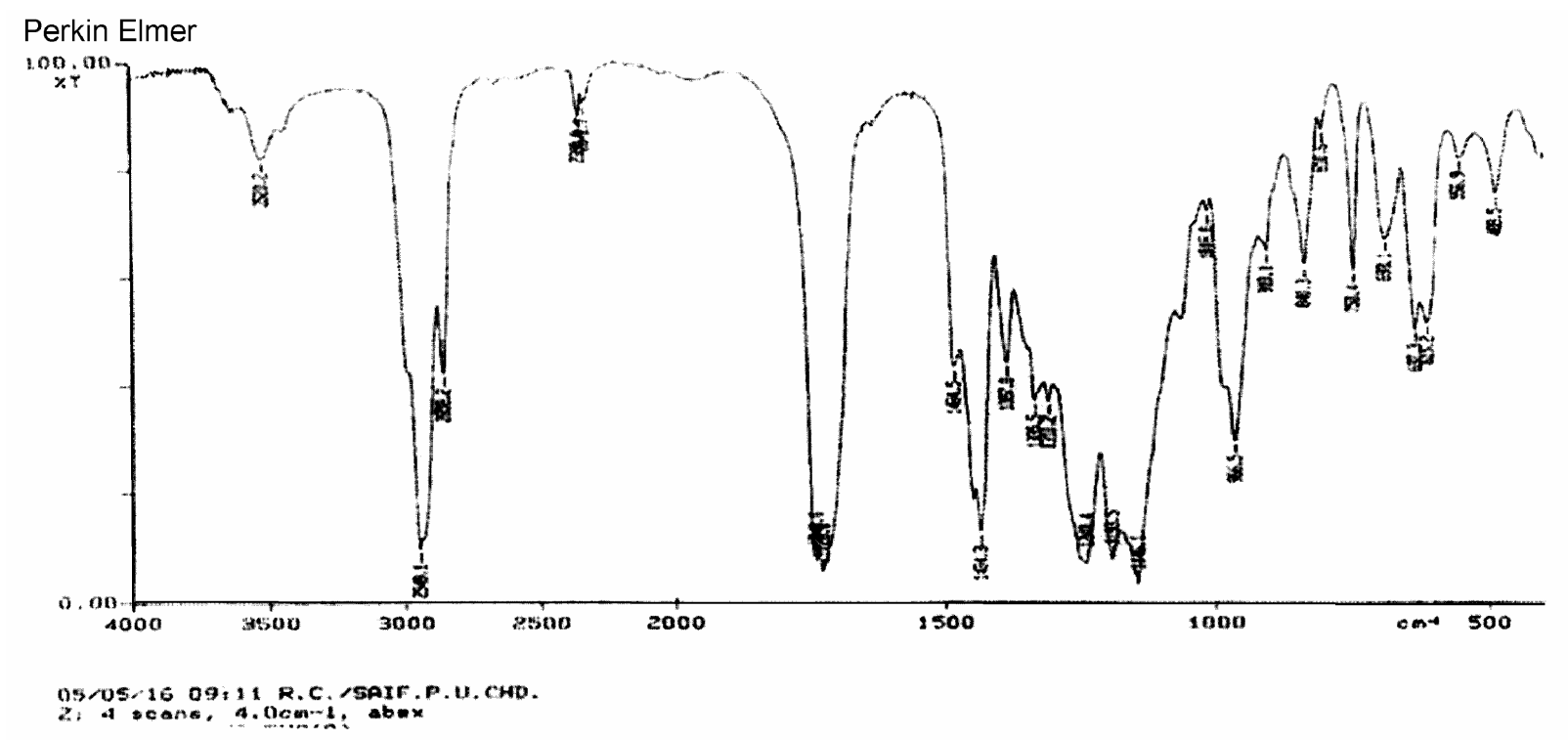

Figure 4. FTIR spectra of PVC/PMMA. 


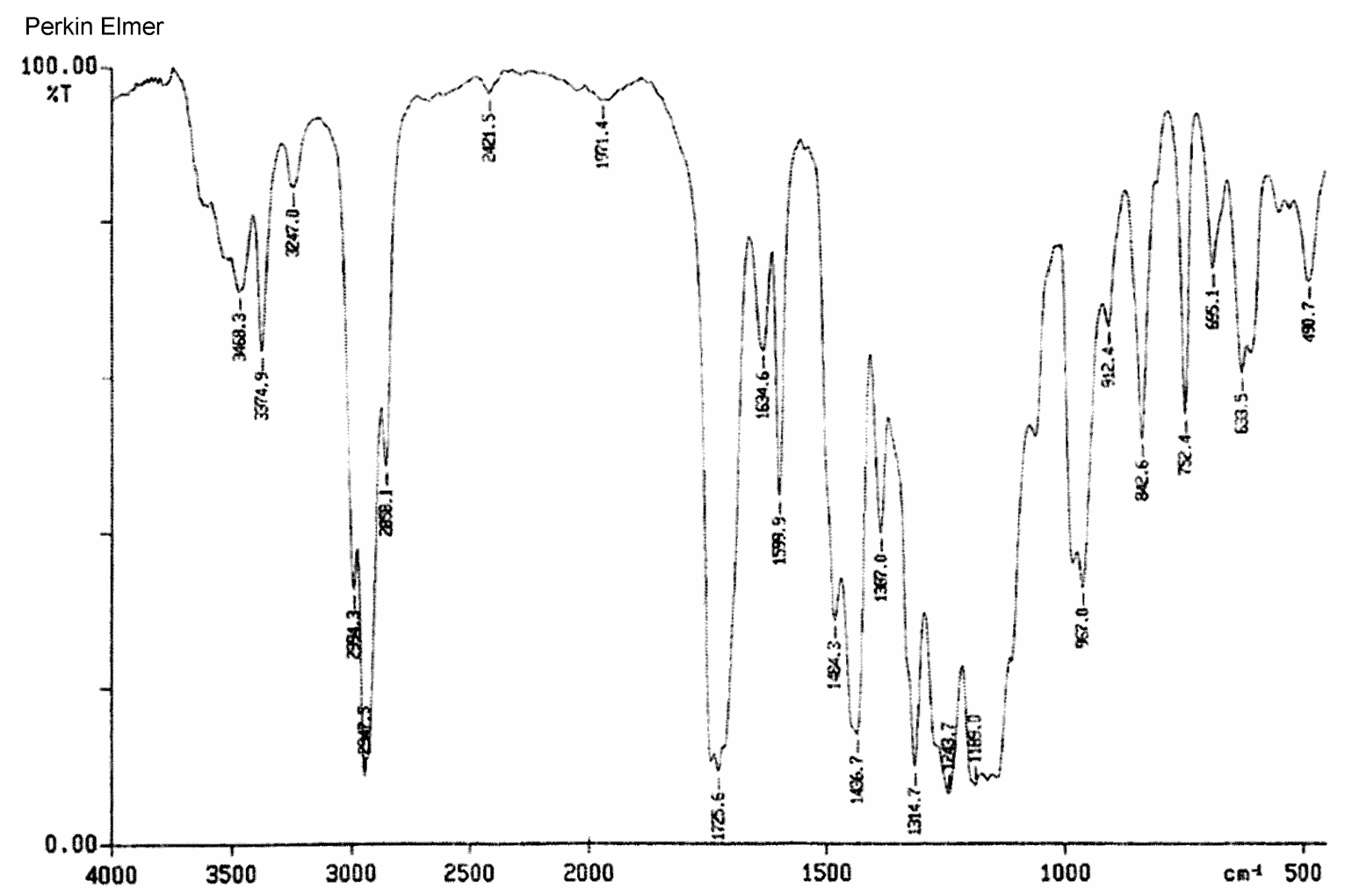

05/09/0? 12:54 R.C./SAIF.P.U.CHD.

$X: 4$ scans, $4.0 \mathrm{~cm}-1$, flat, smooth, abex spl. code : PMMA+PVC+ 4\%PNA

Figure 5. FTIR spectra of PVC/PMMA/4\% PNA.

tion band at $2949 \mathrm{~cm}^{-1}$ in PVC/PMMA blend (figure 4) is broadened in PVC/PMMA $/ 4 \%$ PNA blend and shows absorption peaks at three frequencies. On doping, medium to strong absorption band appears at $1599 \mathrm{~cm}^{-1}$, which is not present in pure PVC/PMMA blend, indicating the presence of $\mathrm{N}-\mathrm{H}$ bonding from $\mathrm{NH}_{2}$ group. The appearance of new frequencies, shifting of absolute frequencies and band broadening in these cases may be due to the change in tacticity and geometry in the structures of the blend. Below frequency, $1100 \mathrm{~cm}^{-1}$, the numbers of absorption peaks get lowered on doping. The lower end frequencies are slightly shifted on doping the blend.

\subsection{XRD study}

XRD (not included in this script) of PVC/PMMA, PVC/ PMMA/4\%PNA, and PVC/PMMA/8\%PNA showed almost an amorphous nature.

\section{Conclusions}

(I) Electrical conductivity, $\sigma\left(\mathrm{S} \mathrm{cm}^{-1}\right)$, of PVC $(7.61 \times$ $10^{-13}-1.04 \times 10^{-10}$ ) has been found greater than that of
PMMA $\left(8.02 \times 10^{-15}-4.31 \times 10^{-12}\right)$ at various temperatures ranging from $313-373 \mathrm{~K}$ and voltages ranging from $25-$ $800 \mathrm{~V}$.

(II) Electrical conductivity, $\sigma\left(\mathrm{S} \mathrm{cm}^{-1}\right)$, of PVC/PMMA polyblend $\left(1.02 \times 10^{-13}-1.11 \times 10^{-11}\right)$ has been found to lie intermediate between that of PVC and PMMA at various temperatures.

(III) The increase in doping concentration of PNA increases the conductivity for PVC, PMMA and PVC/PMMA polyblend. Variation in conductivity of polyblend with respect to dopant concentration (at all temperatures and applied voltages) is less marked as compared to those in individual polymers, PVC and PMMA.

(IV) The dielectric constant of samples increases with the increase of (i) temperature at constant dopant concentration and (ii) dopant concentration at constant temperature.

(V) The activation energies, in high field region for all samples have been found to decrease with increase in electric field.

The addition of dopant PNA to $1: 1$ PVC/PMMA polyblend does not considerably affect the electrical conductivity. This demonstrates that the polyblend is also electrically stable besides its proven thermal stability. PNA adds benzene ring, oxygen and nitrogen atoms to the polyblend 
matrix and is known to increase the tensile strength of the host matrix. Our study leads us to conclude that PVC/ PMMA/4\% PNA polyblend matrix possesses better electrical insulating properties.

\section{Acknowledgements}

Authors are thankful to UGC, Western Region, Pune, for sanctioning the study leave under FIP ( $\mathrm{X}^{\text {th }}$ plan) to one of the authors (RVW). They are also extremely grateful to Dr K N Patil, Principal, V B M V, Amravati, for extending laboratory facilities to carry out the present work.

\section{References}

Bahadur P and Sastry N V 2003 Principles of polymer science (New Delhi: Narosa Publishing House) p. 281

Bahri R and Seth R K 1997 Indian J. Pure \& Appl. Phys. 35104

Belsare N G and Deogaonkar V S 1998a Indian J. Pure \& Appl. Phys. $\mathbf{3 6} 280$
Belsare N G and Deogaonkar V S 1998b Indian J. Pure \& Appl. Phys. 15157

Blythe A R 1979 Electrical properties of polymers (Cambridge: Cambridge University Press) 95 p. 105

Francise A Carey 2003 Organic chemistry (New Delhi: Tata McGraw Hill Publishing Co.) 5th ed., p. 922

Goosey Martin T 1985 Plastics and their properties for electronic and electrical applications (Amsterdam: Elsevier) pp $12-13$

Khare P K, Gaur M S and Srivastava A P 1994 Indian J. Pure \& Appl. Phys. 3214

Purohit G and Joshi G C 2001 Indian J. Phys. B75 571

Rajendran S and Uma T 2000 Mater. Letts 44242

Seanor Donald A 1982 Electrical properties of polymers (New York: Academic Press) pp 7-9, 15-27

Skotheim Terje A 1986 Handbook of conducting polymers (New York: Marcel Dekker Inc) 2 p. 917

Smith William F 1990 Principles of materials science and engineering (New York: McGraw Hill Publishing Company) 2nd ed, pp 331-333

Tager A 1972 Physical chemistry of polymers (Moscow: MIR Publication) p. 282 